Research Article

\title{
Clinical Applicability of Whole-Exome Sequencing Exemplified by a Study in Young Adults with the Advanced Cryptogenic Cholestatic Liver Diseases
}

\author{
Maria Kulecka, ${ }^{1}$ Andrzej Habior, ${ }^{1}$ Agnieszka Paziewska, ${ }^{1}$ Krzysztof Goryca, ${ }^{2}$ \\ Michalina Dabrowska, ${ }^{2}$ Filip Ambrozkiewicz, ${ }^{1}$ Bożena Walewska-Zielecka, ${ }^{3}$ \\ Andrzej Gabriel, ${ }^{4}$ Michal Mikula, ${ }^{2}$ and Jerzy Ostrowski ${ }^{1,2}$ \\ ${ }^{1}$ Department of Gastroenterology, Hepatology and Clinical Oncology, Medical Center for Postgraduate Education, Roentgena 5, \\ 02-781 Warsaw, Poland \\ ${ }^{2}$ Department of Genetics, Cancer Center-Institute, Roentgena 5, 02-781 Warsaw, Poland \\ ${ }^{3}$ Department of Public Health, Faculty of Health Sciences, Medical University of Warsaw, Żwirki i Wigury 61, \\ 02-091 Warsaw, Poland \\ ${ }^{4}$ Department of Pathomorphology, Medical University of Silesia, Medyków 18, 40-752 Katowice, Poland
}

Correspondence should be addressed to Jerzy Ostrowski; jostrow@warman.com.pl

Received 9 December 2016; Accepted 11 April 2017; Published 24 May 2017

Academic Editor: Haruhiko Sugimura

Copyright (C) 2017 Maria Kulecka et al. This is an open access article distributed under the Creative Commons Attribution License, which permits unrestricted use, distribution, and reproduction in any medium, provided the original work is properly cited.

Background. The proper use of new medical tests in clinical practice requires the establishment of their value and range of diagnostic usefulness. While whole-exome sequencing (WES) has already entered the medical practice, recognizing its diagnostic usefulness in multifactorial diseases has not yet been achieved. Aims. The objective of this study was to establish usability of WES in determining genetic background of chronic cholestatic liver disease (CLD) in young patients. Methods. WES was performed on six young patients (between 17 and 22 years old) with advanced fibrosis or cirrhosis due to CLD and their immediate families. Sequencing was performed on an Ion Proton sequencer. Results. On average, 19,673 variants were identified, of which from 7 to 14 variants of an individual were nonsynonymous, homozygous, recessively inherited, and considered in silico as pathogenic. Although monogenic cause of CLD has not been determined, several heterozygous rare variants and polymorphisms were uncovered in genes previously known to be associated with CLD, including ATP8B1, ABCB11, RXRA, and $A B C C 4$, indicative of multifactorial genetic background. Conclusions. WES is a potentially useful diagnostic tool in determining genetic background of multifactorial diseases, but its main limitation results from the lack of opportunities for direct linkage between the uncovered genetic variants and molecular mechanisms of disease.

\section{Introduction}

The pathogenesis of many liver diseases, such as viral hepatitis, is simple and well-known, while others have a more complicated, or still obscure, development. Some hepatobiliary disorders are of strict genetic origin but the majority are more complex and arise from environmental exposure underlined by individual susceptibility. Such complex pathogenesis is frequently observed in cholestatic liver diseases (CLD), in which the risk of liver injury and its clinical presentation depends upon deteriorated hepatic excretion and enterohepatic circulation of bile acids and other cholephiles, directed by alterations in hepatocyte transporters or their transactivators [1-3]. However, there are still patients in whom liver injury must be termed as cryptogenic despite substantial diagnostic efforts.

While single-gene disorders are manifested mostly in childhood, complex disorders typically appear at a later age. Discovery of monogenic disorders, caused by highly penetrant variants, remains within the possibilities of contemporary genetic research. Therefore, hereditary CLD in children, which result from mutations of genes involved 
TABLE 1: Symptoms, laboratory data, and histopathology at presentation in six young patients with cryptogenic chronic cholestatic liver injury. ALP: alkaline phosphatase; TBA: total bile acids; $\gamma$-GT: gamma glutamyl transpeptidase; N: normal; LFT: liver function tests; N/A: not available; BRIC 2: benign recurrent intrahepatic cholestasis; sdPSC: small duct primary sclerosing cholangitis.

\begin{tabular}{|c|c|c|c|c|c|c|c|c|c|}
\hline \multirow{2}{*}{$\begin{array}{l}\text { Patient } \\
\text { number }\end{array}$} & \multirow[t]{2}{*}{ Sex/age } & \multirow[t]{2}{*}{ Symptoms } & \multicolumn{6}{|c|}{ Serum chemistry (x ULN) } & \multirow[t]{2}{*}{ Histology } \\
\hline & & & ALT & AST & ALP & $\gamma-\mathrm{GT}$ & Bilirubin & TBA & \\
\hline 1 & $\mathrm{~F} / 20$ & Fatigue, weight loss & 4 & $\mathrm{~N}$ & 6 & 5.6 & $\mathrm{~N}$ & 6.5 & $\begin{array}{l}\text { Extended fibrosis, with bridging } \\
\text { fibrous septa, scarce inflammatory } \\
\text { activity, focal ductular reaction. }\end{array}$ \\
\hline 2 & $\mathrm{M} / 17$ & $\begin{array}{l}\text { Hepatomegaly, abnormal LFT, } \\
\text { Crohn's disease; portal hypertension }\end{array}$ & 1.4 & $\mathrm{~N}$ & 2.7 & 4.2 & $\mathrm{~N}$ & N/A & $\begin{array}{l}\text { Chronic mild hepatitis with } \\
\text { minimal ductular reaction, } \\
\text { porto-portal fibrosis, possible cirrhosis. } \\
\text { No sdPSC features. }\end{array}$ \\
\hline 3 & $\mathrm{M} / 17$ & Hepatosplenomegaly, no symptoms & 1.8 & 1.2 & 3.9 & 2.8 & $\mathrm{~N}$ & N/A & $\begin{array}{l}\text { Liver cirrhosis, mild chronic } \\
\text { inflammation. }\end{array}$ \\
\hline 4 & $\mathrm{~F} / 22$ & Hepatosplenomegaly, no symptoms & 2 & $\mathrm{~N}$ & 2.5 & 4.5 & $\mathrm{~N}$ & 4 & $\begin{array}{l}\text { Chronic mild hepatitis, bridging } \\
\text { fibrosis; minimal ductular reaction. }\end{array}$ \\
\hline 5 & $\mathrm{M} / 19$ & Hepatosplenomegaly, no symptoms & 2.8 & $\mathrm{~N}$ & 1.4 & 7.1 & $\mathrm{~N}$ & 6.6 & $\begin{array}{l}\text { Liver cirrhosis, mild inflammation, } \\
\text { ductular reaction. }\end{array}$ \\
\hline 6 & $\mathrm{~F} / 22$ & Jaundice, pruritus & 1.5 & $\mathrm{~N}$ & 1.3 & $\mathrm{~N}$ & 3 & N/A & $\begin{array}{c}\text { Extra- and intracellular } \\
\text { bilirubinostasis. Minimal } \\
\text { inflammatory activity, BRIC } 2 .\end{array}$ \\
\hline
\end{tabular}

in bile acid and phosphatidylcholine transport, such as $A T P 8 B 1$ [4, 5], $A B C B 11$ [6], and $A B C B 4$ [2], are relatively easy to diagnose. Conversely, identification of rare, potentially pathogenic variants, which do not segregate in strict Mendelian fashion but contribute individually to disease risk, is still challenging, even if next-generation sequencing (NGS) is employed.

Whole-exome sequencing (WES) allows the analysis of all exons of all protein-coding genes and is considered as a cost-effective technology for detection of disease variants underlying Mendelian disorders, as well as for cataloguing common and rare disease-related genomic alterations [7-10]. However, while the clinical applicability of WES has been extensively discussed [9], the clinicians' point of view is rather rarely presented in the scientific literature.

In this study, we present a WES-based genetic screening, obtained from an academic laboratory, of six young patients and their immediate families. All patients presented advanced chronic CLD of unknown pathogenesis and were candidates for liver transplantation. Our experience highlights a high potential efficiency of the WES approach in uncovering the broad genetic load, but points out a deficiency of the analytical methods which would prove the final biological linkage between identified genetic variant(s) and molecular mechanisms of disease.

\section{Materials and Methods}

2.1. Patients. Six patients, aged 17-22, were referred between 2012 and 2015 to the Department of Gastroenterology and Hepatology at the Medical Centre for Postgraduate Education in Warsaw suffering from advanced cryptogenic CLD. None of these patients showed symptoms of childhood cholestasis or $\alpha 1$-antitrypsin deficiency; cystic fibrosis and autoimmune liver diseases were excluded. At presentation, autoantibodies and markers of $\mathrm{HAV}, \mathrm{HBV}$, and $\mathrm{HCV}$ and also EBV and CMV were not found. Copper and iron metabolism markers and serum IgG4 were normal. No abnormalities of the biliary tree were recorded on MRCP. A liver biopsy was performed in all patients, and histological examination revealed advanced changes - mainly fibrosis or cirrhosis without features of specific liver disease (details in Table 1).

Consent for clinical WES was obtained from the patients and their families. An internal review board approval was obtained from the Medical Centre for Postgraduate Education. The study protocol conforms to the ethical guidelines of the 1975 Declaration of Helsinki (6th revision, 2008) as reflected in a priori approval by the institution's human research committee.

Patient number 1: The patient is a twenty-year-old female born in 1990 to consanguineous parents (mother's and father's grandparents were brothers). She was healthy and a little overweight $\left(\mathrm{BMI}=26.5 \mathrm{~kg} / \mathrm{m}^{2}\right)$. In 2011, she suffered from fatigue and weight loss. In 2009, she was taking two dietary supplements- "organic chromium" (containing chromium maleate and niacin) and "Ha-pantoten classic"-over a period of three months. The patient denied taking other medicines, drugs, or any alcohol consumption. Her brother and parents were healthy.

Patient number 2: Symptoms of inflammatory bowel disease appeared in a previously healthy male patient born in 1993, when he was 17 years old. Abnormal LFT were recorded (ALP, $\gamma$-GT > AST, and ALT) at this time. Crohn's disease of the large bowel was diagnosed, and 5-ASA treatment was started. There was no history of other medical treatment, drugs, dietary supplement usage, or alcohol consumption. The criteria of primary sclerosing cholangitis (PSC) and small duct PSC were not fulfilled. Family history in this patient was not remarkable.

Patient number 3 (born in 1991) and patient number 5 (born in 1990): These two male patients had a similar 
medical history. At 18 and 19 years of age, respectively, they were referred for the first time to the hospital due to hepatomegaly, abnormal LFT (ALP, $\gamma$-GT > AST, and ALT), and low platelet count. The disease course till hospitalization was "silent." No history of other diseases, medical treatment, dietary supplements, drugs, or alcohol had been recorded in these patients. The etiology of the disease was not established and cryptogenic liver cirrhosis was diagnosed. Family history was unremarkable for both patients.

Patient number 4: The patient is an only child born in 1993. Hepatomegaly and abnormal LFT without jaundice and pruritus were observed in her third year of life. A CMV infection was diagnosed and she was put on standard treatment with ganciclovir without changes in LFT. Due to sustained laboratory abnormalities (ALP and $\gamma$-GT $>$ AST and ALT), she was under observation for several years in a pediatric hospital but the cause of liver disease was not found despite an extensive diagnostic approach. The patient's mother was healthy but her father died from undiagnosed liver disease when he was 40 years old (material for any genetic study was unavailable). The patient denied cigarette smoking, alcohol consumption, taking medicines, or dietary supplements.

Patient number 6: The patient is a 22 -year-old female born in 1989 suffered from itching, who became icteric during her second month of using oral contraceptives. There was no history of jaundice in her neonatal period or childhood. The clinical picture, laboratory data (low activity of $\gamma$-GT), and liver histology were suggestive of benign recurrent intrahepatic cholestasis type 2 (BRIC 2), but genetic testing which could have confirmed this was not performed at the time. The patient's parents and two siblings were healthy.

2.2. Exome Sequencing. Genomic DNA was extracted from the whole blood treated with EDTA using a QIAamp DNA Mini Kit. A human exome sequencing library was prepared using the Ion AmpliSeq ${ }^{\mathrm{TM}}$ Exome Kit (Thermo Fisher) according to the manufacturer's protocol. Briefly, $100 \mathrm{ng}$ of genomic DNA was subjected to multiplex amplification with 2x Exome Primer Pool. Next, primers were digested and adapters were ligated to the amplicons. The samples were then purified using Agencourt AMPure XP beads (Beckman Coulter) and stored at $-20^{\circ} \mathrm{C}$ for further processing. The concentration of each library was measured using a Qubit fluorometer (Thermo Fisher), and DNA fragment length was measured using High Sensitivity DNA Analysis Kits on a Bioanalyzer 2100 (Agilent). Each library was diluted to $\sim 100 \mathrm{pM}$ prior to template preparation. Up to three barcoded libraries were subjected to automated template preparation with an Ion PI IC 200 Kit on the Ion Chef Instrument, which performs emulsion PCR on ion sphere particles, followed by particle recovery and template loading on a PI chip. Samples were sequenced in an Ion Proton instrument on a PI chip using the sequencing reagents provided as part of the Ion PI IC $200 \mathrm{Kit}$, according to the manufacturer's instructions.

2.3. Read Mapping and Variant Calling. Raw reads were processed by the Torrent Suite analysis pipeline and mapped to human genome assembly hg19 by TMAP. Variant calls were made by two programs: VarScan [11] (with the trio calling option when the family trio was available) and Torrent Variant Caller. The detailed parameters for running both of these programs are included in Supplementary Material Online File 1 available online at https://doi.org/ $10.1155 / 2017 / 4761962$. Additionally, single-nucleotide variant calls were filtered by a variant-filter [12]. If multiple alleles were reported on one position, they were separated by vcfbreakmulti script from vcflib [13].

2.4. Consanguinity Verification. In order to verify possible consanguinity, the numbers of shared rare variants were compared. With the use of a python script, one variant from each chromosome (except for the $\mathrm{Y}$ chromosome) present in the father was randomly chosen. This variant list was then compared to variants present in the mother, and the ratio of the expected number of variants shared by the mother and father (based on 1000 genomes minor allele frequency for Europe) and the observed number of such variants was computed. This procedure was repeated 100 times for variants with minor allele frequency smaller than $0.01,0.02,0.05$, and 0.1 . This procedure was conducted on the variants from VarScan family trio calling. In order to compare the in-family odds-ratio to the population ratio, the father samples were also compared between families.

2.5. Variant Annotation. Variant annotation, including such information as gene, variant consequence, in silico prediction of deleteriousness by SIFT [14] and PolyPhen [15], and various minor allele frequencies, was performed by the variant effect predictor [16]. Variants located in noncoding regions and synonymous variants were excluded from further analysis, unless it was predicted they might affect splicing.

2.6. Variant Filtering and Gene Prioritization. The list of variants was narrowed down by python script to variants in genes which are involved in

(a) bile acid and bile salt metabolism, according to the Reactome database [17];

(b) bile secretion, according to the KEGG database [18];

(c) CLD, according to the MalaCards database [19];

(d) liver diseases, according to the MalaCards database;

(e) lipid metabolism, according to the Reactome database.

This data were retrieved from these databases in June 2014. Full gene lists are provided in Supplementary Material Online File 2.

If family trios were available, the following variants were taken separately into consideration:

(a) Variants inherited in autosomal recessive manner

(b) Variants inherited in autosomal dominant manner

(c) Compound heterozygous variants. 
TABLE 2: Summary of variants present in coding regions in every available member of family trio (on the left) and of recessive homozygous variants (on the right) if the full family trio was available.

\begin{tabular}{|c|c|c|c|c|c|c|c|c|}
\hline \multicolumn{2}{|c|}{ Patient and family } & Variants & SNP & Indels & Recessive homozygotes & Rare & Nonsynonymous & Deleterious in silico \\
\hline \multirow{3}{*}{ Number 1} & Father & 19,477 & 18,849 & 538 & 832 var & 54 & 26 & 13 \\
\hline & Mother & 19,211 & 18,540 & 574 & 7 indel & & & \\
\hline & Child & 19,856 & 19,216 & 568 & 825 SNP & & & \\
\hline \multirow{3}{*}{ Number 2} & Father & 17,104 & 16,617 & 426 & 789 var & 30 & 17 & 9 \\
\hline & Mother & 20,200 & 19,640 & 500 & 7 indel & & & \\
\hline & Child & 20,051 & 19,499 & 477 & 782 SNP & & & \\
\hline \multirow{3}{*}{ Number 3} & Father & 17,406 & 16,842 & 504 & 719 var & 27 & 15 & 4 \\
\hline & Mother & 19,500 & 18,920 & 505 & 5 indel & & & \\
\hline & Child & 20,313 & 19,685 & 543 & 714 SNP & & & \\
\hline \multirow{2}{*}{ Number 4} & Mother & 20,184 & 19,668 & 478 & & & & \\
\hline & Child & 18,738 & 18,253 & 441 & & & & \\
\hline \multirow{2}{*}{ Number 5} & Mother & 20,325 & 19,746 & 519 & & & & \\
\hline & Child & 18,480 & 17,922 & 497 & & & & \\
\hline \multirow{3}{*}{ Number 6} & Father & 21,021 & 20,541 & 440 & 862 var & 31 & 15 & 7 \\
\hline & Mother & 21,520 & 20,974 & 499 & 2 indel & & & \\
\hline & Child & 21,377 & 20,827 & 500 & 860 SNP & & & \\
\hline Mean & & 19,673 & 19,109 & 501 & & & & \\
\hline
\end{tabular}

These variants were chosen using a python script. Gene prioritization was conducted in Endeavour [20], with genes previously associated with hereditary cholestatic syndromes $A T P 8 B 1, A B C B 11$, and $A B C B 4$ as training genes.

\section{Results}

Average coverage in targeted regions in the six patients ranged from $36 \mathrm{x}$ to $77 \mathrm{x}$ (median: 60x). The percentage of bases with coverage more than $20 \mathrm{x}$ ranged from $57 \%$ to $80 \%$ (median: 72\%). The detailed statistics, including average coverage in main genes connected with familiar cholestasis ( $A T P 8 B 1, A B C B 11$, and $A B C B 4$ ) are presented in Supplementary Material Online, Table S1.

To reduce the rate of uncertain findings, the family trio exome sequencing has been recommended [21]. In our research, we had access to both parents of four affected probands and only mothers of the other two. On average, there were 19,673 variants in coding regions discovered per person: 19,091 SNPs and 501 indels. Each child possesses on average 800 recessive homozygous variants, out of which between 27 and 55 variants are rare variants, 15 and 27 are nonsynonymous, and 7 and 14 are deleterious in silico (i.e., are classified as deleterious by either SIFT or PolyPhen score, or their IMPACT in variant effect predictor is high). Only one variant was present in more than one sample: FAM104B p.Arg111Ter, present in patient numbers 2 and 3. However, $F A M 104 B$ is a gene with a currently unknown function, so no further causal link could be established (Table 2, Supplementary Material Online, Table S2). Gene prioritization was conducted for genes in which recessive homozygous potentially deleterious variants were present, but the top five genes after prioritization, ABCB5, SKD2, DUS3L, HDAC10, and $L M F 2$, have no direct link to liver function or bile acid metabolism as far as we know (Supplementary Material Online, Table S3). Therefore, we decided to scan lists of genes mentioned in Materials and Methods in order to identify variants which may lead to susceptibility to cholestatic liver injury.

Variants were not reported if found as homozygous in any of the unaffected parents. Only two rare heterozygous missense variants (with minor allele frequency [MAF] smaller than 1\%) were present within genes connected with hereditary cholestatic syndromes (such as ATP8B1 [22], $A B C B 11$ [23], and $A B C B 4$ [24], as well as TJP2, BAAT [25], CYP7A1 [26], CYP7B1 [27], HSD3B7 [28], and AKR1D1 [29]). These were variants in ATP8B1: p.Asn45Thr and p.Ile349Thr in patient numbers 2 and 3 , respectively (Table 3 A). Variant p.Asn45Thr was previously found in European patient cohorts with cholestasis and cholestasis of pregnancy [30]. Moreover, a novel nonsense variant was uncovered in patient number 6-ABCB11 p.Ser25Ter. In addition, all but two (patient numbers 5 and 6) affected subjects possessed $A B C B 11$ p Ala444Val polymorphism; patient numbers 1,2 , and 3 were homozygous for this variant. However, since this polymorphism is very common (GMAF $=0.408$ in 1000 genomes), it was not possible to establish a causative link between this variant and CLD in our patients. This polymorphism was previously described as associated with intrahepatic cholestasis of pregnancy [31, 32].

Two mutations in AKR1C1 p.Arg170His and RXRA p.Pro22Leu, genes involved in bile acid metabolism (according to the Reactome databases), were found in patient number 5, and a mutation in ABCC4 p.Lys304Asn, a gene involved in bile acid transport and secretion, was uncovered in patient number 6 (Table $3 \mathrm{~B}, \mathrm{C}$ ). Four polymorphisms associated with drug metabolism, CYP2C19 c.681G >A(p.=), CYP2C9 p.Ile359Leu, NAT2 p.Ile114Thr, 
TABLE 3: Variants in genes connected with the following: (A) cholestatic liver diseases, (B) bile acid metabolism, (C) bile acid transport and secretion, and (D) other genes of significance, including genes connected with other liver diseases and lipid metabolism.

\begin{tabular}{|c|c|c|c|c|}
\hline Family & $\mathrm{A}$ & B & $\mathrm{C}$ & $\mathrm{D}$ \\
\hline Number 1 & & & & $\begin{array}{l}\text { CYP2C19, c.681G }>\text { A, } \\
\text { CYP2C9 p.Ile359Leu, } \\
\text { NAT2, p.Ile114Thr } \\
\text { NAT2, p.Arg197Gln }\end{array}$ \\
\hline Number 2 & $\begin{array}{l}\text { ATP8B1 p.Asn } 45 \mathrm{Thr} \text {, } \\
0 \mid 1 \text { in patient and father }\end{array}$ & & & KRT8, p.Ile91Val \\
\hline Number 3 & $\begin{array}{c}\text { ATP8B1p.Ile349Thr, } \\
0 \mid 1 \text { in patient and mother }\end{array}$ & & & \\
\hline \multicolumn{5}{|l|}{ Number 4} \\
\hline Number 5 & & $\begin{array}{c}A K R 1 C 1, \text { p.Arg170His } \\
0 \mid 1 \text { in patient } \\
R X R A \text {, p.Pro22Leu } \\
0 \mid 1 \text { in patient and mother }\end{array}$ & & \\
\hline Number 6 & $\begin{array}{c}A B C B 11 \text { p.Ser25Ter } \\
0 \mid 1 \text { in patient and father }\end{array}$ & & $\begin{array}{c}\text { ABCC4 p.Lys304Asn } \\
0 \mid 1 \text { in patient and mother }\end{array}$ & PEMT p.Arg226Trp \\
\hline
\end{tabular}

TABLE 4: Variant characteristics, including global minor allele frequency (GMAF) in 1000 genomes project, European American minor allele frequency (EA MAF) in NHLBI exome sequencing project (ESP), and deleteriousness prediction according to SIFT and PolyPhen are provided.

\begin{tabular}{lcccc}
\hline Gene & Amino acid change & Rs & GMAF/EA MAF & SIFT/PolyPhen \\
\hline ATP8B1 & p.Asn45Thr & rs146599962 & $0.0016 / 0.0043$ & Tolerated_low_confidence/benign \\
ATP8B1 & p.Ile349Thr & rs56214207 & $(-) / 0.0002$ & Tolerated/benign \\
AKR1C1 & p.Arg170His & rs139588200 & $0.0028 / 0.0083$ & Tolerated/benign \\
ABCC4 & p.Lys304Asn & - & - & Deleterious/benign \\
RXRA & p.Pro22Leu & rs55836231 & $0.0004 / 0.0014$ & Tolerated_low_confidence/benign \\
CYP2C19 & c.681G>A(p.=) & rs4244285 & $0.2214 / 0.1484$ & $-/-$ \\
CYP2C9 & p.Ile359Leu & rs1057910 & $0.0485 / 0.1094$ & Deleterious/possibly_damaging \\
NAT2 & p.Arg197Gln & rs1799930 & $0.2650 /-$ & Deleterious/possibly_damaging \\
NAT2 & p.Ile114Thr & rs1801280 & $0.2927 / 0.3466$ & Deleterious/possibly_damaging \\
\hline
\end{tabular}

and NAT2 p.Arg197Gln, were found in patient number 1, and a heterozygous variant KRT8 p.Ile91Val (this gene was previously found as associating with cryptogenic cirrhosis) was found in patient number 2 (Table $3 \mathrm{D}$ ). The heterozygous variant PEMT p.Arg226Trp (in a gene responsible for synthesis of phosphatidylcholine in liver) was found in patient number 6. All variants were heterozygous and are further characterized and described in Table 4.

Among missense mutations in genes involved in PFIC (ATP8B1) (Table $3 \mathrm{~A}$ ) or connected with bile secretion and metabolism (AKR1C1, ABCC4, and RXRA) (Table $3 \mathrm{~B}, \mathrm{C}$ ), none were predicted to be deleterious by either SIFT or PolyPhen (Table 4). However, patient number 1 possessed four polymorphisms connected with drug metabolism. Patient number 2 possessed a rare variant in the gene connected with other liver diseases: KRT8 (cryptogenic cirrhosis). Patient number 6 possessed a rare variant in PEMT, a gene responsible for the synthesis of phosphatidylcholine in the liver. All these variants were predicted to be deleterious by both SIFT and PolyPhen (Table 4).
In consanguinity verification, the ratio between expected and observed number of very rare variants was very high in family number 1 (88.95); it is extremely unusual to observe such a ratio in the wider population (Figure 1), indicating that the parents might be related to each other. Using WES, we uncovered previously unreported consanguineous relations in this family: it was revealed that the parents shared a common great-grandfather.

\section{Discussion}

A genetic diagnostic is not particularly difficult if the uncovered gene has been previously implicated in a similar condition, but identifying novel disease genes is often challenging [33, 34]. While 7000 known rare diseases are reported by the National Institutes of Health Office of Rare Diseases Research, each specific genetic disease can be considered to be rare. Up to 25-30 million people and $25 \%$ of pediatric patients in the United States may be affected by a 


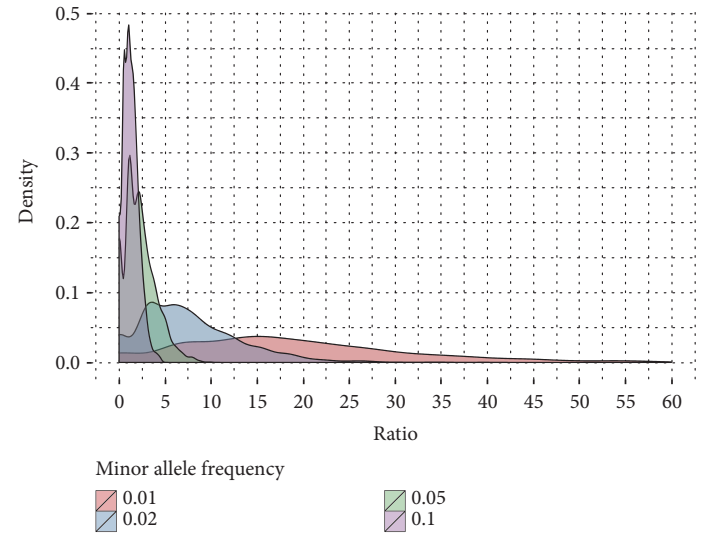

FIGURE 1: Distribution of ratios between observed and expected number of rare variants, measured for variants with EUR MAF lesser than $0.01,0.02,0.05$, and 0.1 measured between available fathers in sequenced families.

rare genetic disease [34]. If so, many rare diseases remain undiagnosed [35].

Alcohol consumption, viral hepatitis $\mathrm{B}$ and $\mathrm{C}$, and nonalcoholic fatty liver disease (NAFLD) represent the most frequent chronic liver diseases leading to cirrhosis and primary liver cancer [4]. Autoimmune hepatitis and various forms of CLD are less frequent causes of progressive liver diseases. Metabolic and hereditary CLD, for example, biliary atresia, Alagille syndrome, congenital hepatic fibrosis, and progressive familial intrahepatic cholestasis (PFIC), are typical in infancy and childhood and may progress over a few years to liver failure $[5,6]$. Other metabolic disorders, such as $\alpha 1$-antitrypsin deficiency and cystic fibrosis, are also present during the early years of life, but their progress to cirrhosis is slower. In adults, the most common progressive CLD are primary biliary cholangitis (PBC) and primary sclerosing cholangitis (PSC) [36].

Hereditary cholestatic syndromes in children, clinically presenting as progressive familial intrahepatic cholestasis (PFIC) types $1-3$, result from mutations of $A T P 8 B 1[4,5]$, $A B C B 11$ [6], and $A B C B 4$ [2]. More than 100 mutations in $A B C B 11$, which encodes the bile salt export pump (BSEP), have been recognized to date. These genetic defects may be responsible for morphological changes in the liver in some patients, progressing to cirrhosis and even hepatocellular carcinoma [2,37]. Mutations in ATP8B1 or ABCB4 (multidrug resistance transporter-MDR3) that lead to minor functional limitations only, cause less severe liver injury. Other polymorphisms, especially in the $A B C B 11$ and $A B C B 4$ genes, are usually clinically harmless [38]. Their clinically relevant cholestatic syndromes, similar to those related to the V444A polymorphism of BSEP, are in combination with acquired triggers such as pregnancy or exposure to drugs or toxins $[3,39,40]$. Various other forms of hereditary cholestasis (such as Aagenaes syndrome, North American Indian childhood cirrhosis, cirhin deficiency, Dubin-Johnson syndrome, and Alagille syndrome) may result from rare mutations of genes encoding bile acid synthesis enzymes (CYP7A1 [26], CYP7B1 [27], HSD3B7 [28], and AKR1D1 [29]), the bile acid receptor farnesoid X receptor (FXR), the Notch signaling pathway, sodium taurocholate cotransporting polypeptide (NTCP), or tight junction proteins along the canalicular membrane of hepatocytes and cholangiocytes $[2,4,5]$. In turn, drug-induced liver injury is the most commonly acquired cholestatic disease in adults [3]. In some cases the acute form of drug-induced liver injury may progress to chronic liver disease [41]. Other chronic cholestatic diseases seen in adults are PBC and PSC; both are autoimmune polygenic disorders with genetic load represented by common, mostly non-protein-coding SNPs (single-nucleotide polymorphisms) of a small effect size [4]. Thus, although several pathogenic mutations responsible for molecular mechanism of the disease have been found in children and some young patients with inherited CLD [2, 39-41], the pathogenesis of acquired CLD is more complicated.

Of the six studied patients, patient numbers 1 and 4 did not possess any rare variant in the genes connected with cholestatic diseases or bile salt secretion and metabolism. In turn, patient number 1 possessed four polymorphisms connected with drug metabolism (CYP2C19 c.681G >A(p.=), CYP2C9 p.Ile359Leu, NAT2 p.Ile114Thr, and NAT2 p.Arg197Gln), out of which CYP2C9 p.Ile359Leu is of particular importance since it leads to impaired metabolism of widely available nonsteroidal anti-inflammatory drugs $[42,43]$. Patient numbers 2 and 3 possessed rare, heterozygous variants in ATP8B1: p.Ile349Thr and p.Asn45Thr, respectively; the latter has been found previously by others to be associated with cholestasis [30]. In addition, patient number 2 possessed another deleterious variant: KRT8 p.Ile91Val. This variant is predicted to be deleterious by at least one algorithm, while other mutations in KRT8 have been connected with the development of various liver diseases, including biliary atresia and cirrhosis $[44,45]$. Patient number 6 possessed a heterozygous nonsense variant $A B C B 11$ p. Ser25Ter. The nonsense variant, even heterozygous, is likely to contribute to a lower level of ABCB11 protein. Moreover, this patient possessed another deleterious variant: PEMT p.Arg226Trp which could predispose to the development of liver disease in the early years of life. Four patients (numbers 1,2,3, and 6) possessed a common polymorphism $A B C B 11$ p.Ala444Val (three of them were homozygous for this variant), which in pregnant women or after exposure to hepatotoxic drugs or toxins may associate with intrahepatic cholestasis of pregnancy $[31,32]$ and other cholestatic syndromes $[3,39,40]$. Patient number 6 also possessed another located on this same gene. Thus, all the uncovered variants, especially in patient numbers 1,2 , and 6 , may contribute to cholestasis, probably in combination with external triggers. Unfortunately, we cannot submit to this assumption any evidence from a functional study, and lack of similar studies in the literature precludes any comparative analysis.

\section{Conclusions}

While WES can be applied for diagnostic setting and as a discovery tool [10], its diagnostic yield depends on the age of disease onset, the presence of a positive family history, 
and specific clinical phenotypes, and its success rate for identifying rare causative variants reaches $15-30 \%$ [10, 33, 34]. WES allows the cataloguing of genes enriched for rare variants, but, perhaps more importantly, we need effective methods to investigate the heritability of specific functional alleles in complex disorders. The unresolved question is how to separate the individual causative effects of rare alleles with low/moderate penetrance from the effect of polygenic burden of common variants. For discovery of rare incompletely penetrant variants, even large studies are underpowered due to the enormous number of affected subjects needed to establish significance of the association, where more than half of the approximately 7.5 million variants found by the exome sequencing of 60,706 individuals have been seen only once [46]. Therefore, although WES has already been integrated into healthcare with the possibility of uncovering exceedingly rare conditions [46], the proper use of WES in clinical practice still requires new tools for both identification of causative variants among existing incidental findings and variants of unknown/uncertain significance (VUS) and selection of patients most likely to benefit from high throughput genetic testing $[9,10]$. In other words, we need standards for interpretation of variant complexity, resulting from a collaborative effort between physicians and biologists, who provide clinical and experimental data, and computational biologists, who describe the molecular phenotype of disease.

Lack of independent verification restrains WES-based studies at the early stages of clinical applicability. For a better WES clinical setting, we need more complete and less biased databases composed of variants associating with well-characterized phenotypes which should pursue maximum data dissemination in the community [9, 47]. When more and more WES data is combined with medical records from various clinical situations, data interpretation may improve, and the proper use of WES in clinical practice could become a reality.

\section{Conflicts of Interest}

The authors declare no competing interest.

\section{Acknowledgments}

This research is supported by 2011/01/B/NZ5/05291 grant (for Andrzej Habior) and 2011/02/A/NZ5/00339 grant (for Jerzy Ostrowski) from the National Science Centre.

\section{References}

[1] M. Wagner and M. Trauner, "Recent advances in understanding and managing cholestasis," F1000Research, vol. 5, 2016.

[2] G. M. Hirschfield, "Genetic determinants of cholestasis," Clinics in Liver Disease, vol. 17, no. 2, pp. 147-159, 2013.

[3] C. G. Dietrich and A. Geier, "Effect of drug transporter pharmacogenetics on cholestasis," Expert Opinion on Drug Metabolism \& Toxicology, vol. 10, no. 11, pp. 1533-1551, 2014.

[4] M. Blachier, H. Leleu, M. Peck-Radosavljevic, D.-C. Valla, and F. Roudot-Thoraval, "The burden of liver disease in Europe: a review of available epidemiological data," Journal of Hepatology, vol. 58, no. 3, pp. 593-608, 2013.

[5] S. C. Ling, "Congenital cholestatic syndromes: what happens when children grow up?" Canadian Journal of Gastroenterology, vol. 21, no. 11, pp. 743-751, 2007.

[6] A. Davit-Spraul, E. Gonzales, C. Baussan, and E. Jacquemin, "Progressive familial intrahepatic cholestasis," Orphanet Journal of Rare Diseases, vol. 4, no. 1, p. 1, 2009.

[7] K. Panoutsopoulou, I. Tachmazidou, and E. Zeggini, "In search of low-frequency and rare variants affecting complex traits," Human Molecular Genetics, vol. 22, no. R1, pp. R16R21, 2013.

[8] P. L. Auer and G. Lettre, "Rare variant association studies: considerations, challenges and opportunities," Genome Medicine, vol. 7, no. 1, p. 16, 2015.

[9] G. Bertier, M. Hétu, and Y. Joly, "Unsolved challenges of clinical whole-exome sequencing: a systematic literature review of end-users' views," BMC Medical Genomics, vol. 9, no. 1, p. 52, 2016.

[10] L. G. Biesecker and R. C. Green, "Diagnostic clinical genome and exome sequencing," The New England Journal of Medicine, vol. 370, no. 25, pp. 2418-2425, 2014.

[11] D. C. Koboldt, K. Chen, T. Wylie et al., "VarScan: variant detection in massively parallel sequencing of individual and pooled samples," Bioinformatics, vol. 25, no. 17, pp. 22832285, 2009.

[12] C. Kandoth, D. Koboldt, and D. Larson, "Variant-filter," July 2015, https://github.com/ckandoth/variant-filter.

[13] E. Garrison, "Vcflib: a C++ library for parsing and manipulating VCF files," July 2015, https://github.com/ekg/vcflib.

[14] P. C. Ng and S. Henikoff, "SIFT: predicting amino acid changes that affect protein function," Nucleic Acids Research, vol. 31, no. 13, pp. 3812-3814, 2003.

[15] I. A. Adzhubei, S. Schmidt, L. Peshkin et al., "A method and server for predicting damaging missense mutations," Nature Methods, vol. 7, no. 4, pp. 248-249, 2010.

[16] W. McLaren, B. Pritchard, D. Rios, Y. Chen, P. Flicek, and F. Cunningham, "Deriving the consequences of genomic variants with the Ensembl API and SNP Effect Predictor," Bioinformatics, vol. 26, no. 16, pp. 2069-2070, 2010.

[17] D. Croft, A. F. Mundo, R. Haw et al., "The Reactome pathway knowledgebase," Nucleic Acids Research, vol. 42, no. D1, pp. D472-D477, 2014.

[18] M. Kanehisa, S. Goto, Y. Sato, M. Kawashima, M. Furumichi, and M. Tanabe, "Data, information, knowledge and principle: back to metabolism in KEGG," Nucleic Acids Research, vol. 42, no. D1, pp. D199-D205, 2014.

[19] N. Rappaport, M. Twik, N. Nativ et al., "MalaCards: a comprehensive automatically-mined database of human diseases," Current Protocols in Bioinformatics, vol. 47, no. 1.24, pp. 1.24.1-1.24.19, 2014.

[20] L.-C. Tranchevent, R. Barriot, S. Yu et al., "ENDEAVOUR update: a web resource for gene prioritization in multiple species," Nucleic Acids Research, vol. 36, Supplement 2, pp. W377-W384, 2008.

[21] P. S. Atwal, M.-L. Brennan, R. Cox et al., "Clinical wholeexome sequencing: are we there yet?" Genetics in Medicine, vol. 16, no. 9, pp. 717-719, 2014.

[22] L. W. Klomp, L. N. Bull, A. S. Knisely et al., "A missense mutation in FIC1 is associated with Greenland familial cholestasis," Hepatology, vol. 32, no. 6, pp. 1337-1341, 2000. 
[23] S. S. Strautnieks, L. N. Bull, A. S. Knisely et al., "A gene encoding a liver-specific $\mathrm{ABC}$ transporter is mutated in progressive familial intrahepatic cholestasis," Nature Genetics, vol. 20, no. 3, pp. 233-238, 1998.

[24] J. M. de Vree, E. Jacquemin, E. Sturm et al., "Mutations in the MDR3 gene cause progressive familial intrahepatic cholestasis," Proceedings of the National Academy of Sciences of the United States of America, vol. 95, no. 1, pp. 282-287, 1998.

[25] V. E. H. Carlton, B. Z. Harris, E. G. Puffenberger et al., "Complex inheritance of familial hypercholanemia with associated mutations in TJP2 and BAAT," Nature Genetics, vol. 34, no. 1, pp. 91-96, 2003.

[26] C. R. Pullinger, C. Eng, G. Salen et al., "Human cholesterol 7alpha-hydroxylase (CYP7A1) deficiency has a hypercholesterolemic phenotype," The Journal of Clinical Investigation, vol. 110, no. 1, pp. 109-117, 2002.

[27] K. D. Setchell, M. Schwarz, N. C. O'Connell et al., "Identification of a new inborn error in bile acid synthesis: mutation of the oxysterol 7alpha-hydroxylase gene causes severe neonatal liver disease," The Journal of Clinical Investigation, vol. 102, no. 9, pp. 1690-1703, 1998.

[28] M. S. Buchmann, E. A. Kvittingen, H. Nazer et al., "Lack of 3 beta-hydroxy-delta 5-C27-steroid dehydrogenase/isomerase in fibroblasts from a child with urinary excretion of 3 betahydroxy-delta 5-bile acids. A new inborn error of metabolism," The Journal of Clinical Investigation, vol. 86, no. 6, pp. 2034-2037, 1990.

[29] K. D. Setchell, F. J. Suchy, M. B. Welsh, L. Zimmer-Nechemias, J. Heubi, and W. F. Balistreri, "Delta 4-3-oxosteroid 5 betareductase deficiency described in identical twins with neonatal hepatitis. A new inborn error in bile acid synthesis," The Journal of Clinical Investigation, vol. 82, no. 6, pp. 21482157, 1988.

[30] M. Langhirt, M. Krawczyk, S. Weber, and F. Lammert, "Intrahepatic cholestasis: analysis of genetic variants in an European cohort," Zeitschrift für Gastroenterologie, vol. 50, no. 8, 2012.

[31] P. H. Dixon, S. W. C. van Mil, J. Chambers et al., "Contribution of variant alleles of ABCB11 to susceptibility to intrahepatic cholestasis of pregnancy," Gut, vol. 58, no. 4, pp. 537-544, 2009.

[32] Y. Meier, T. Zodan, C. Lang et al., "Increased susceptibility for intrahepatic cholestasis of pregnancy and contraceptiveinduced cholestasis in carriers of the 1331T $>$ C polymorphism in the bile salt export pump," World Journal of Gastroenterology, vol. 14, no. 1, pp. 38-45, 2008.

[33] X. Zhu, S. Petrovski, P. Xie et al., "Whole-exome sequencing in undiagnosed genetic diseases: interpreting 119 trios," Genetics in Medicine, vol. 17, no. 10, pp. 774-781, 2015.

[34] K. D. Farwell, L. Shahmirzadi, D. El-Khechen et al., "Enhanced utility of family-centered diagnostic exome sequencing with inheritance model-based analysis: results from 500 unselected families with undiagnosed genetic conditions," Genetics in Medicine, vol. 17, no. 7, pp. 578-586, 2015.

[35] V. Shashi, A. McConkie-Rosell, B. Rosell et al., "The utility of the traditional medical genetics diagnostic evaluation in the context of next-generation sequencing for undiagnosed genetic disorders," Genetics in Medicine, vol. 16, no. 2, pp. 176-182, 2014.

[36] K. Boonstra, U. Beuers, and C. Y. Ponsioen, "Epidemiology of primary sclerosing cholangitis and primary biliary cirrhosis: a systematic review," Journal of Hepatology, vol. 56, no. 5, pp. 1181-1188, 2012.
[37] A. S. Knisely, S. S. Strautnieks, Y. Meier et al., "Hepatocellular carcinoma in ten children under five years of age with bile salt export pump deficiency," Hepatology, vol. 44, no. 2, pp. 478-486, 2006.

[38] J. C. Chambers, W. Zhang, J. Sehmi et al., "Genome-wide association study identifies loci influencing concentrations of liver enzymes in plasma," Nature Genetics, vol. 43, no. 11, pp. 1131-1138, 2011.

[39] E. Pasmant, P. Goussard, L. Baranes et al., "First description of ABCB4 gene deletions in familial low phospholipidassociated cholelithiasis and oral contraceptives-induced cholestasis," European Journal of Human Genetics, vol. 20, no. 3, pp. 277-282, 2012.

[40] S. S. Strautnieks, J. A. Byrne, L. Pawlikowska et al., "Severe bile salt export pump deficiency: 82 different ABCB11 mutations in 109 families," Gastroenterology, vol. 134, no. 4, pp. 1203-1214, 2008.

[41] J. G. Stine and N. Chalasani, "Chronic liver injury induced by drugs: a systematic review," Liver International, vol. 35, no. 11, pp. 2343-2353, 2015.

[42] J. A. G. Agúndez, C. Martínez, D. Pérez-Sala, M. Carballo, M. J. Torres, and E. García-Martín, "Pharmacogenomics in aspirin intolerance," Current Drug Metabolism, vol. 10, no. 9, pp. 998-1008, 2009.

[43] J. A. G. Agúndez, E. García-Martín, and C. Martínez, "Genetically based impairment in CYP2C8- and CYP2C9dependent NSAID metabolism as a risk factor for gastrointestinal bleeding: is a combination of pharmacogenomics and metabolomics required to improve personalized medicine?" Expert Opinion on Drug Metabolism \& Toxicology, vol. 5, no. 6, pp. 607-620, 2009.

[44] N. O. Ku, R. Gish, T. L. Wright, and M. B. Omary, "Keratin 8 mutations in patients with cryptogenic liver disease," The New England Journal of Medicine, vol. 344, no. 21, pp. 1580-1587, 2001.

[45] N. O. Ku, J. M. Darling, S. M. Krams et al., "Keratin 8 and 18 mutations are risk factors for developing liver disease of multiple etiologies," Proceedings of the National Academy of Sciences of the United States of America, vol. 100, no. 10, pp. 6063-6068, 2003.

[46] J. E. Posey, J. A. Rosenfeld, R. A. James et al., "Molecular diagnostic experience of whole-exome sequencing in adult patients," Genetics in Medicine, vol. 18, no. 7, pp. 678$685,2016$.

[47] J. D. Jongbloed, A. Pósafalvi, W. S. Kerstjens-Frederikse, R. J. Sinke, and J. P. van Tintelen, "New clinical molecular diagnostic methods for congenital and inherited heart disease," Expert Opinion on Medical Diagnostics, vol. 5, no. 1, pp. 9-24, 2011. 


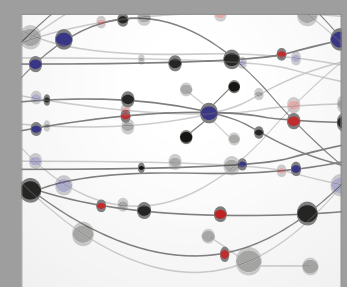

The Scientific World Journal
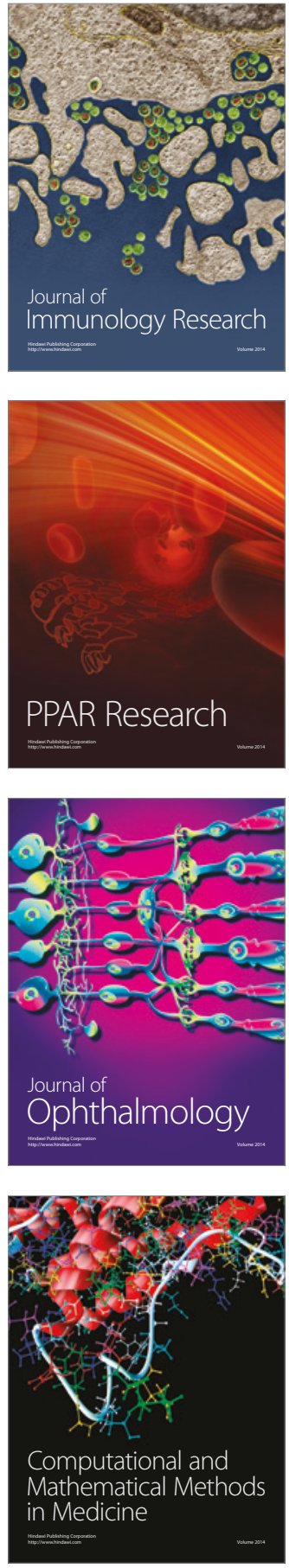

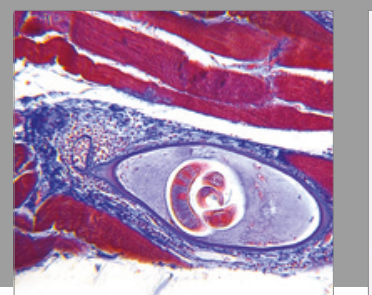

Gastroenterology Research and Practice
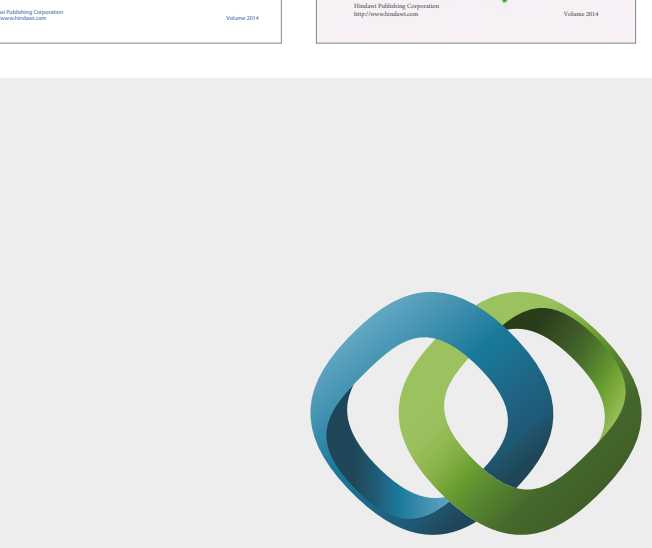

\section{Hindawi}

Submit your manuscripts at

https://www.hindawi.com
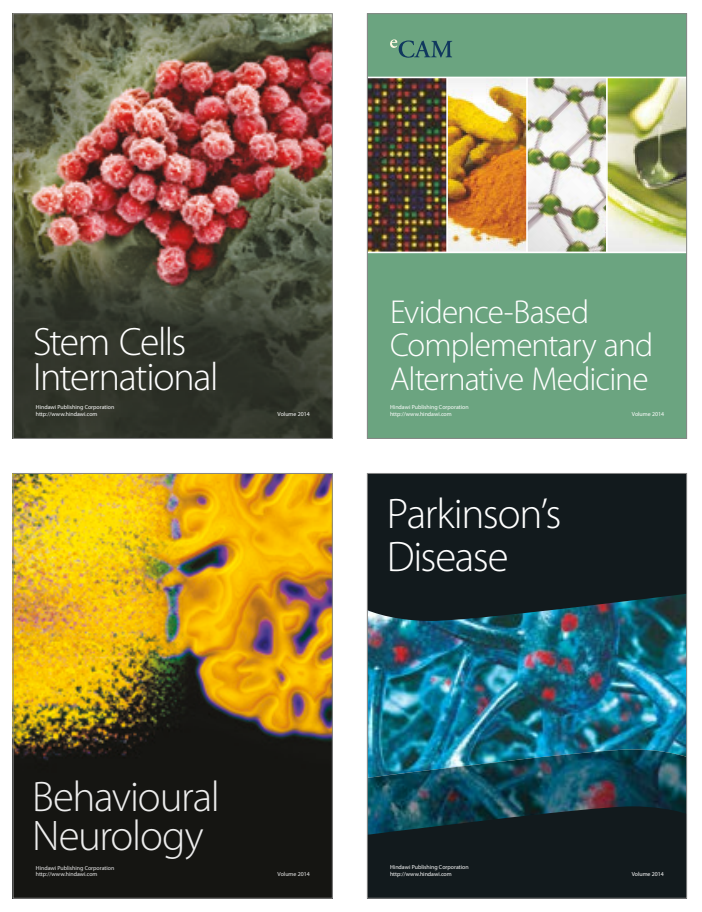
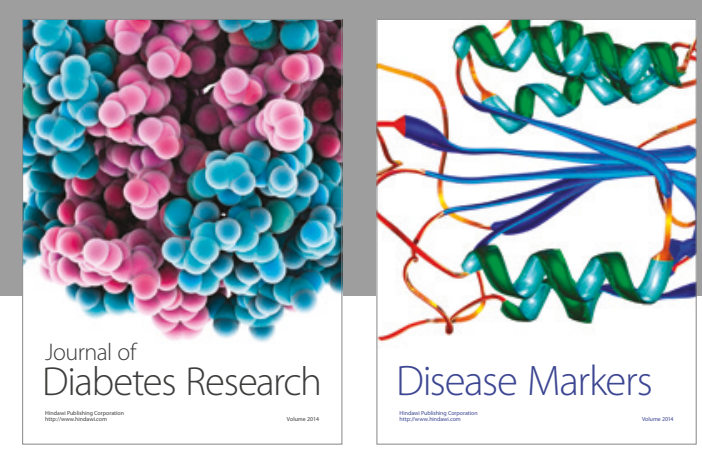

Disease Markers
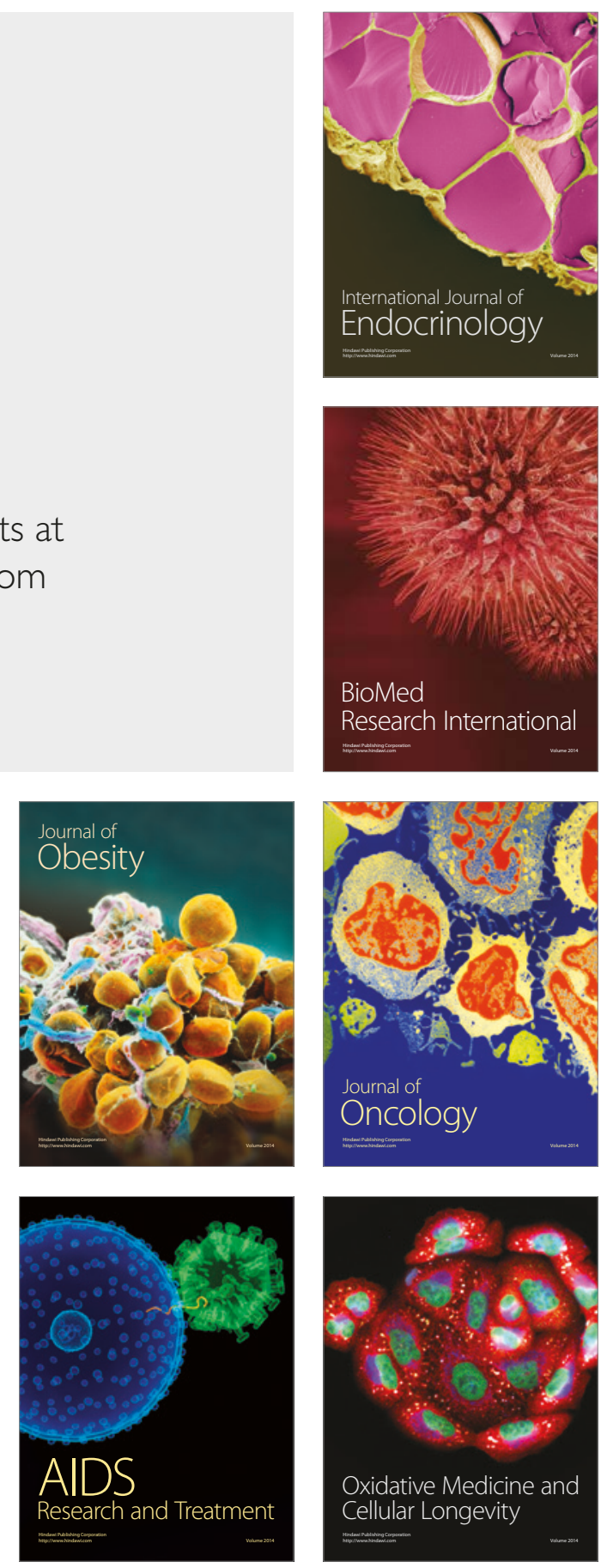